\title{
Evaluation of Anticoagulant Monitoring in Pediatric Patients Receiving Enoxaparin for Treatment of Venous Thrombosis
}

Jason Koury, PharmD; Robert Hellinga, PharmD; Jennifer Rose, BSPH; Shirley Abraham, MD; and Anjali Subbaswamy, MD

\begin{abstract}
OBJECTIVES A venous thromboembolism (VTE) is a blood clot that occurs secondary to vessel wall injury often from a central line insertion. Enoxaparin is often considered a first-line treatment in pediatrics for VTE due to its favorable kinetic profile. Enoxaparin monitoring for pediatric patients is accomplished through anti-Xa monitoring in which monitoring practices may vary between institutions. The objective of this study is to evaluate covariates in pediatric patients to determine which variables are most likely to be associated with enoxaparin dose changes as a result of anti-Xa monitoring.
\end{abstract}

METHODS A single center, retrospective chart review was conducted in pediatric patients treated with enoxaparin for VTE over a 10-year period and who were assessed to determine covariates that lead to dose changes based on anti-Xa levels. Secondary outcomes described monitoring patterns at the University of New Mexico Children's Hospital.

RESULTS Sixty-eight patients met inclusion criteria in which results showed that patients aged 2 to 5.9 months $(p=0.026)$, who had critical care status $(p=0.009)$, and who were of Native American ethnicity $(p=0.016)$ were likely to have an enoxaparin dose change at least once during their treatment regimen. The mean number of levels drawn were 7.5 per patient over a 6- to 12-week period, and doses were not frequently changed based on a confirmatory lab draw. However, many doses were adjusted based on the week 1 post-therapeutic level.

CONCLUSIONS Patients of Native American ethnicity, younger than 6 months, and those admitted to the PICU were likely to have dose changes based on anti-Xa levels.

ABBREVIATIONS AKI, acute kidney injury; BMI, body mass index; CHEST, American College of Chest Physicians; DVT, deep vein thrombosis; ICU, intensive care unit; PE, pulmonary embolism; PICU, pediatric intensive care unit; VTE, venous thromboembolism

KEYWORDS enoxaparin; low molecular weight heparin; monitoring; venous thromboembolism

J Pediatr Pharmacol Ther 2021;26(4):346-351

DOI: 10.5863/1551-6776-26.4.346

\section{Introduction}

A venous thromboembolism (VTE) is a blood clot that encompasses a deep vein thrombosis (DVT) or pulmonary embolism (PE), which leads to various signs, symptoms, and tissue destruction based on clot location. This usually occurs after injury to a vessel from central line insertion, trauma, surgery, and acute or chronic disease states that initiate the intrinsic or extrinsic coagulation cascade. Enoxaparin, a low molecular weight heparin, is considered the standard of care for VTE treatment in children due to greater predictability of kinetics and the ability to monitor levels. ${ }^{1}$ It works by binding to and accelerating antithrombin III activity and therefore inhibiting coagulation factor Xa and lla. Through Xa inhibition, the conversion of prothrombin to thrombin is inhibited, which leads to the prevention of a fibrin clot formation. Venous thromboembolism treatment varies based on many factors such as age, weight, renal function, and treatment indication.

According to the Centers for Disease Control and Prevention data from 1994 and 2009, 78,685 pediatric patients were discharged with a VTE diagnosis, of which 3740 were associated with an in-hospital death. ${ }^{2}$ The annual incidence of VTE in children is 0.7 to 2.1 per 100,000 people and added an average cost of $\$ 25,000$ per child per year. ${ }^{3}$ These costs may include, but are not limited to, length of stay, diagnostic tests, treatment, hospitalizations, outpatient visits, and comorbidity management. ${ }^{4}$

Enoxaparin therapeutic monitoring for pediatric patients is accomplished through anti-Xa monitoring with a goal of 0.5 to $1.0 \mathrm{IU} / \mathrm{mL}$. At our institution, this level is usually drawn on average of 4 hours (3-6 hours) after the second or third dose of initiation or a new dosing regimen. Once a stable level is obtained then a repeat level is drawn 4 hours after the following dose. Traditionally in adult patients, anti-Xa monitoring is not routinely per- 
formed, mainly due to a potential of incorrectly drawing the levels and pharmacokinetic and pharmacodynamic stability in adult patients. ${ }^{5-7}$ Monitoring may prove to be beneficial in adults if they have renal dysfunction or obesity. Studies have yet to demonstrate a true correlation between anti-Xa levels and enoxaparin efficacy or safety, especially in children. ${ }^{8-10}$ Hence, it is difficult to understand the full utility of monitoring of all children on enoxaparin therapy, which may lead to unnecessary health care costs.

The frequency of levels being drawn varies based on health care team's comfort level, especially when a therapeutic value is obtained. Currently, the only source that directs the number of times a level is drawn after a therapeutic level is based on expert opinion, which is established by the American College of Chest Physicians (CHEST) guidelines. ${ }^{1}$ The recommendations are to check levels after starting a dose or dose change and once a correct dose is established based on the anti-Xa levels. It is then recommended to confirm using a repeat level (confirmatory level), then check the following week and monthly once stable. The number of dose adjustments from the CHEST guideline initial dosing and dose adjustment guidelines have shown variability in adjustments in the pediatric population. ${ }^{11}$ To our knowledge, a study has not yet evaluated the characteristics that are associated with enoxaparin dose changes post anti-Xa levels in pediatric patients. The objective of this study is to evaluate covariates in pediatric patients to determine which variables are most likely to be associated with enoxaparin dose changes, post anti-Xa levels, which will lead to more predictable anticoagulant management.

\section{Materials and Methods}

Study Population and Data Collection. This study was approved by the Institutional Review Board at University of New Mexico Hospitals with waiver of informed consent. A single center, retrospective chart review was conducted in which pediatric patients who met inclusion criteria who were treated with enoxaparin for VTE (including PE or DVT) over a 10-year period (2009-2019) were assessed. Patients were identified by the International Classification of Diseases (ICD)- $9^{12}$ or ICD-10 ${ }^{13}$ codes for VTE, PE, or DVT. Patients were included if they were 2 months to 18 years of age who had received an enoxaparin dose based on the CHEST guidelines for treatment of VTE and anti-Xa levels drawn to establish target attainment. Patients were excluded if they were neonates, on renal replacement therapy, prescribed enoxaparin for VTE prophylaxis, incomplete data for chart review, or patients who were not managed based on the CHEST guideline dosing recommendations. The primary objective of the study was to determine which characteristics led to enoxaparin dose changes in pediatric patients. The secondary objective was to determine the mean number of dosing changes needed to reach target anti-Xa level, the

\section{Table 1. Analyzed Variables}

Primary Outcomes
Age
Sex
Weight
Ethnicity
Critical care status
Acute kidney injury
Secondary Outcomes
Average starting dose
Average number of dose changes
Average number of levels drawn
Dose changes based on week 1, monthly levels

mean number of anti-Xa levels drawn, and explore the number of dose changes based on the confirmatory at week 1 , and month 1 anti-Xa levels. See Table 1 for variables analyzed. A confirmatory level is defined as a level drawn a day after a therapeutic level is achieved to ensure accuracy.

Study data were collected and managed using REDCap electronic data capture tools hosted at the University of New Mexico. ${ }^{14}$ REDCap is a secure, webbased application designed to support data capture for research studies, providing 1) an intuitive interface for validated data entry; 2 ) audit trails for tracking data manipulation and export procedures; 3) automated export procedures for seamless data downloads to common statistical packages; and 4) procedures for importing data from external sources.

Statistical Analysis. All patients with ICD-9 or ICD-10 codes for VTE, PE, or DVT were included in the analysis. The following demographic data were gathered for patients who met the inclusion criteria: age, sex, ethnicity, weight at enoxaparin initiation (if given, otherwise most recent weight, in kilograms), height (in centimeters), $\mathrm{BMI}$, serum creatinine at initiation of enoxaparin, and critical care status. The following anticoagulation data were gathered for patients meeting inclusion criteria: enoxaparin dose $(\mathrm{mg} / \mathrm{kg})$, anti-Xa levels, and number of enoxaparin dose changes. Data were analyzed using the statistical software, SPSS Statistics for Windows, version 19.0 (IBM Corporation, Armonk, NY). The primary outcome was analyzed using a multiple logistic regression, and secondary outcomes were reported through descriptive statistics.

\section{Results}

Patients. A total of 68 charts, for patients who were admitted to the hospital with an initial VTE, met inclusion criteria. Patient demographics are summarized in Table 2. All patients were initially dosed using the CHEST guidelines. ${ }^{111}$ Of the 68 patients, 37 were male and 31 were female. Most patients were Hispanic followed by Caucasian then Native American. Many of the patients 
Table 2. Demographics, Clinical Characteristics and Multivariate Analysis

\begin{tabular}{|c|c|c|c|}
\hline Variable & Patients, n (\%) & p value & OR \\
\hline \multicolumn{4}{|l|}{ Age group } \\
\hline $2-5.9 \mathrm{mo}$ & $8(12)$ & 0.026 & \\
\hline 6-11.9 mo & $10(15)$ & 0.99 & 0.00 \\
\hline $1-9.9 \mathrm{yr}$ & $20(30)$ & 0.81 & 1.49 \\
\hline$>10 \mathrm{yr}$ & $30(44)$ & 0.08 & 35.02 \\
\hline \multicolumn{4}{|l|}{ Sex } \\
\hline Female & $31(46)$ & 0.43 & 1.90 \\
\hline \multicolumn{4}{|l|}{$\mathrm{BMI}$} \\
\hline $5 \%-84 \%$ & $2(3)$ & 0.40 & \\
\hline $\mathrm{N} / \mathrm{A},<2 \mathrm{yr}$ & $20(30)$ & 0.11 & 22.61 \\
\hline$<5 \%$ & $22(32)$ & 0.99 & 0.00 \\
\hline $85 \%-95 \%$ & $7(10)$ & 0.79 & 1.43 \\
\hline$>95 \%$ & $17(25)$ & 0.10 & 5.68 \\
\hline \multicolumn{4}{|l|}{ Ethnicity } \\
\hline Caucasian & $23(34)$ & 0.19 & \\
\hline Hispanic & $30(44)$ & 0.07 & 0.15 \\
\hline Native American & $13(19)$ & 0.02 & 0.08 \\
\hline African American & $1(1)$ & 1.00 & 1.40 \\
\hline Other & $1(1)$ & 1.00 & 3.29 \\
\hline \multicolumn{4}{|l|}{ Critical care status* } \\
\hline Yes & $23(34)$ & 0.009 & 0.63 \\
\hline \multicolumn{4}{|l|}{ Acute kidney injury ${ }^{+}$} \\
\hline Yes & $5(7)$ & 0.665 & 2.53 \\
\hline
\end{tabular}

were older than a year (74\%), in which more than half of those were $>10$ years. One third of the patients were considered critical care status, defined as admittance to the PICU at the start of enoxaparin therapy. Only 7\% of patients had acute kidney injury (AKI), defined as a creatinine increase by greater than 0.3 from baseline, more than 1.5 to 1.9 times the baseline value, or urine output $<0.5 \mathrm{~mL} / \mathrm{kg} / \mathrm{hr}$ for 6 to 12 hours at the start of the first enoxaparin dose. ${ }^{15}$

Dose Changes. Characteristics that led to enoxaparin dose changes post anti-Xa levels are depicted in Table 2. Results of the multiple logistic regression analysis showed that patients 2 to 5.9 months of age ( $p \leq 0.05)$, who had critical care status $(p \leq 0.05)$ and were of Native American ethnicity ( $\leq 0.05)$ were more likely to have an enoxaparin dose change at least once during their treatment regimen that was guided by anti-Xa levels. It is important to note that 2 of the 13 Native American patients were also admitted to the critical care unit. Characteristics generally regarded as factors that likely will lead to more frequent dose changes such as age 6 to 11.9 months, AKI, and BMI $>$ 95th percentile were not statistically significant. The average starting dose of enoxaparin was $1.1 \mathrm{mg} / \mathrm{kg} /$ dose, and the average number of dose changes were about 2 per patient (Table 3).
Table 3. Dose Range Characteristics

\begin{tabular}{lc} 
Variable & Median (Range) \\
$\begin{array}{l}\text { Starting enoxaparin dose, } \\
\mathrm{mg} / \mathrm{kg} / \mathrm{dose}\end{array}$ & $1.1(0.6-2.6)$ \\
Enoxaparin dose changes & $4(0-11)$ \\
$\begin{array}{l}\text { Anti-Xa serum } \\
\text { concentrations performed }\end{array}$ & $9(1-36)$ \\
\hline
\end{tabular}

Anti-Xa Levels. Due to the retrospective nature of this study, not all anti-Xa levels were drawn consistently. The confirmatory, week 1 , and month 1 anti-Xa levels were correctly obtained in 45,52 , and 52 patients. Of these, $39 / 45$ (87\%) and 44/52 (85\%) patients did not require a dose change based on confirmatory and month 1 levels. A total of 33/52 (63\%) of patients required a dose change based on the week 1 level. Additionally, the average number of levels drawn were 7.5 per patient, during a 6- to 12-week treatment period.

\section{Discussion}

In this retrospective study, we found that there were a few clinical characteristics that correlated with an enoxaparin dosing change based on anti-Xa levels 
for VTE treatment. These characteristics included an age of 2 to 5.9 months, Native American ethnicity, and critical care status, which was defined as admittance to the PICU at the start of enoxaparin. Interestingly, factors such as ages of 6 to 11.9 months, obesity, and AKI did not significantly increase the likelihood of having dose changes after starting enoxaparin. The lack of statistical significance may be due to the low number of patients in our study. Over the 9-year period, only 68 patients met inclusion criteria, which reflects a low incidence seen in pediatric populations. ${ }^{16,17}$

Secondary outcomes focused on enoxaparin monitoring practices. We found that pediatric patients receiving enoxaparin had an average of 7.5 serum anti-Xa concentration, but only received an average of 2 dose changes during a course of therapy, therefore indicating a potential unnecessary amount of testing. Per CHEST guidelines, children with a provoked clot should be treated for 6 to 12 weeks. ${ }^{1}$ Having 7.5 anti-Xa levels checked in that period is approximately $1 \mathrm{blood}$ draw weekly to every other week. Also, many of these patients start their treatment in the hospital as it has been reported that the incidence of hospital acquired VTE is 30 episodes per 10,000 admissions. ${ }^{18}$ Anti-Xa monitoring is more accessible in hospitalize patients, which can sometimes lead to unwarranted monitoring Once a patient is discharged, they must then return for laboratory testing, which can be costly and difficult for families who are in rural communities or those who have a history of non-adherence with appointments.

If one excludes obese patients and those with renal dysfunction, anti-Xa levels are not commonly monitored in the adult population as adult patients tend to have predictable pharmacokinetic. Lee et al ${ }^{19}$ evaluated the need to monitor anti-Xa levels in 99 obese adult patients receiving treatment enoxaparin. They concluded that for safety and potential efficacy purposes, anti-Xa should be monitored in this patient population, although anti-Xa monitoring needs validation with regard to clinical outcomes. In a case series, Ahuja et al ${ }^{20}$ noted that the first anti-Xa level in underweight and renally impaired adult patients was subtherapeutic and often required dose increases to achieve appropriate values. Although it is not considered standard of practice, the authors recommended these, and it is necessary to monitor anti-Xa levels during enoxaparin treatment in these unique adult populations. Although we did not see a statistical significance for obesity or renal impairment leading to dose changes in our population, we strongly recommend continuing to check anti-Xa levels in these patients.

Enoxaparin management is difficult in the pediatric population because dosing is not standardized as it is in adults. Doses are often started at $1 \mathrm{mg} / \mathrm{kg}$ twice a day but may vary based on age. ${ }^{1,11}$ Although all patients begin on a standard dose, it is thought that infants $\leq 6$ months of age require a larger weight-based doses than other age groups and that doses in infants 6 months to 12 months are slightly larger than children 1 year to 18 years. ${ }^{11}$ Variability in enoxaparin dosing necessitates monitoring of anti-Xa levels. Based on our study, it is likely that anti-Xa levels are measured too often in a population that does not always require monitoring. Standard practice has evolved to checking the first level before the second or third dose, a confirmatory level once stable, a 1-week level then a 1-month level and so on. Some practices may even check more often, which leads to higher health care costs and unnecessary blood draws.

In addition to age considerations, it is important to consider the clinical condition when assessing anti-Xa monitoring needs. In a highly inflammatory condition, such as sepsis, a depletion of antithrombin III can occur due to its destruction by neutrophil proteases. ${ }^{21}$ This could be a contributing factor to why patients in a critical care setting require more dose adjustments and monitoring.

A unique and interesting finding of our study was the statistically significant correlation between increased dose changes and Native American ethnicity. Unfortunately, there is very little primary literature available on enoxaparin use in the Native American population. Although Native Americans accounted for $19 \%$ of our total results, it is important to consider that in the Native American sample there were 2/13 patients admitted to the ICU in which multiple factors could have led to dose changes, including having a critical care status. One possible explanation for a need for larger dose changes could be pharmacokinetic or pharmacogenomic differences between ethnic groups. Published data to support this hypothesis are not sufficient but if so could lead to an increase in dosing changes. ${ }^{22,23}$

In order to ensure the safe and effective use of enoxaparin, it is important to consider that some patients should still be monitored more frequently. Included in this list are those who met the primary outcome such as critical care status, Native American ethnicity, and younger than 6 months of age. Although not detected in analysis of our data, for safety purposes, we would recommend more frequent monitoring of anti-Xa levels in patients younger than 10 years old, those with $\mathrm{AKI}$, underweight, obese, or with a clinical judgment by the treating practitioner.

That being said, in these subpopulations one could consider decreasing the number of tests drawn, one of which is the confirmatory test. Based on our results, it may be more cost-effective and important to test after a week of a therapeutic level since it was found that $37 \%$ of our patients required a dose change after a week compared with $13 \%$ after the confirmatory level.

The first limitation to this study is the small sample size being evaluated and the lack of standardization of anti-Xa monitoring. If there were a larger sample, there may have been more data to recommend when 
to test versus not. Due to the infrequent VTE incidence in pediatrics, this could have been more adequate as a multicenter study. Second, this is a retrospective study, hence there are many individualized factors that could not be controlled. Although difficult to conduct, future multi-institutional prospective studies could provide beneficial information on dosing, monitoring, and treatment outcomes of enoxaparin use in the pediatric population. Lastly, the pediatric population is so variable with continuously changing kinetics and dynamics; additionally, growth and development play an important role on how medications are dosed and monitored. Future studies should be focused on starting with a well-defined population that share similar characteristics. A focused approach can provide robust details that can eventually be expanded to the greater population.

\section{Conclusion}

In conclusion, we found that patients of Native American ethnicity, younger than 6 months, and those admitted to the PICU were likely to have dose changes based on anti-Xa levels. Secondarily, it was noted that levels were potentially drawn too often without clinical benefit. An abundance of monitoring increases health care costs and is inconvenient for the patient without an essential clinical benefit. By checking fewer anti-Xa levels, health care costs and patient inconvenience is decreased.

Further prospective studies are warranted to better risk stratify which children require the multiple anti-Xa levels currently checked on all patients. We believe there is clinical equipoise to conduct a prospective study comparing the methods of checking 2 levels (before the third dose and then at 1 week) versus checking our current standard 4 levels, which in the end becomes an average of 7.5 levels checked for 2 dose changes for a 6 - to 12-week treatment course. Due to the variability of practices across institutions, anti-Xa monitoring would be best utilized with a standard practice by drawing levels when it is necessary to implement a therapeutic change.

\section{Article Information}

Affiliations. Department of Inpatient Pharmacy (JK, RH), University of New Mexico Hospitals, Albuquerque, NM; University of New Mexico College of Pharmacy (JR), University of New Mexico, Albuquerque, NM; Department of Pediatric Hematology and Oncology (SA), University of New Mexico, Albuquerque, NM; Department of Pediatrics Critical Care (AS), University of New Mexico, Albuquerque, NM.

Correspondence. Jason, Koury, PharmD, BSN; jaskoury@ salud.unm.edu

Disclosure. The authors declare no conflicts or financial interest in any product or service mentioned in the manuscript, including grants, equipment, medications, employment, gifts, and honoraria. The authors had full access to all data and take responsibility for the integrity and accuracy of the data analysis.

Ethical Approval and Informed Consent. Given the nature of this study, the project was exempt from institution review board/ethics committee review and informed consent or patient assent was not obtained.

Submitted. July 28,2020

Accepted. January 3, 2021

Copyright. Pediatric Pharmacy Association. All rights reserved. For permissions, email: mhelms@pediatricpharmacy.org

\section{References}

1. Monagle P, Chan AKC, Goldenberg NA, et al. Antithrombotic therapy in neonates and children: Antithrombotic Therapy and Prevention of Thrombosis, 9th ed: American College of Chest Physicians Evidence-Based Clinical Practice Guidelines. Chest. 2012;141(suppl 2):e737Se801S.

2. Boulet SL, Amendah D, Grosse SD, Hooper WC. Health care expenditures associated with venous thromboembolism among children. Thromb Res. 2012;129(5):583587.

3. Fernandez MM, Hogue S, Preblick R, Kwong WJ. Review of the cost of venous thromboembolism. Clinicoecon Outcomes Res. 2015;7:451-462.

4. Kufel WD, Seabury RW, Darko W, et al. Clinical feasibility of monitoring enoxaparin anti-Xa concentrations: are we getting it right? Hosp Pharm. 2017;52(3):214-220.

5. Stutsky ME, Reardon DP, Lee Al. Clinical practice patterns of anti-Xa monitoring during low molecular weight heparin therapy. Blood. 2015;126(23):3275. doi 10.1182/ blood.V126.23.3275.3275

6. Garcia DA, Baglin TP, Weitz JI, et al. Parenteral anticoagulants: Antithrombotic Therapy and Prevention of Thrombosis, 9th ed: American College of Chest Physicians Evidence-Based Clinical Practice Guidelines. Chest. 2012;141(2 Suppl):e24S-e43S.

7. Leung $\mathrm{M}, \mathrm{Ho} \mathrm{SH}$, Hamilton DP, et al. Utility of antiXa monitoring in children receiving enoxaparin for therapeutic anticoagulation. J Pediatr Pharmacol Ther. 2005;10(1):43-50.

8. Rosenbloom D, Ginsberg JS. Arguments against monitoring levels of anti-factor $\mathrm{Xa}$ in conjunction with lowmolecular weight heparin therapy. Can J Hosp Pharm. 2002;55(1):15-19.

9. Bounameaux H, De Moerloose P. Is laboratory monitoring of low-molecular-weight heparin therapy necessary? No. J Thromb Haemost. 2004;2(4):551-554.

10. Enoxaparin. Lexi-Drugs. Lexicomp. Riverwoods, IL: Wolters Kluwer Health, Inc. Accessed August 3, 2019. http:// online.lexi.com

11. Dinh CN, Moffett BS, Galati M, et al. A critical evaluation of enoxaparin dose adjustment guidelines in children. J Pediatr Pharmacol Ther. 2019;24(2):128-133.

12. World Health Organization. International Classification of Diseases, Ninth Revision. Accessed December 2019, https://icdlist.com/icd-9/look-up 
13. World Health Organization. International Classification of Diseases, Tenth Revision. Accessed December 2019, https://icd.who.int/browse10/2019/en

14. Harris PA, Taylor R, Thielke R, et al. Research electronic data capture (REDCap): a metadata-driven methodology and workflow process for providing translational research informatics support. J Biomed Inform. 2009;42(2):377-381.

15. Khwaja A. KDIGO clinical practice guidelines for acute kidney injury. Nephron Clin Pract. 2012;120(4):c179-c184.

16. van Ommen $\mathrm{CH}$, Heijboer $\mathrm{H}$, Büller $\mathrm{HR}$, et al. Venous thromboembolism in childhood: a prospective two-year registry in The Netherlands. J Pediatr. 2001;139(5):676-681.

17. Tuckuviene R, Christensen AL, Helgestad J, et al. Pediatric venous and arterial noncerebral thromboembolism in Denmark: a nationwide population-based study. J Pediatr. 2011;159(4):663-669.

18. Takemoto CM, Sohi S, Desai K, et al. Hospital-associated venous thromboembolism in children: incidence and clinical characteristics. J Pediatr. 2014;164(2):332-338.

19. Lee YR, Vega JA, Duong HN, et al. Monitoring enoxaparin with antifactor Xa levels in obese patients. Pharmacotherapy. 2015;35(11):1007-1015.

20. Ahuja T, Mousavi KM, Klejmont L, et al. Enoxaparin dosing and antiXa monitoring in specialty populations: a case series of renal-impaired, extremes of body weight, pregnant, and pediatric patients. PT. 2018;43(10):609-614.

21. Opal SM, Kessler CM, Roemisch J, Knaub S. Antithrombin, heparin, and heparan sulfate. Crit Care Med. 2002;30(5):S325-S331.

22. Jaja C, Burke W, Thummel K, et al. Cytochrome p450 enzyme polymorphism frequency in indigenous and Native American populations: a systematic review. Community Genet. 2008;11(3):141-149.

23. Murphy JE, Severnak T. Gentamicin pharmacokinetics in Native Americans of Apache ancestry. Am J Health Syst Pharm. 1996;53(18):2189-2191. 